\title{
Extragonadal Embryonal Carcinoma
}

National Cancer Institute

\section{Source}

National Cancer Institute. Extragonadal Embryonal Carcinoma. NCI Thesaurus. Code C8880.

An embryonal carcinoma that develops as a primary tumor in an anatomic site other than the testis or ovary. 